\title{
ACERCA DE LA ETNOARQUEOLOGÍA EN AMÉRICA DEL SUR*
}

\author{
Gustavo G. Politis \\ Universidad Nacional del Centro de la Provincia de Buenos Aires y \\ Universidad Nacional de La Plata - Argentina
}

Resumen: La Etnoarqueología es una disciplina de desarrollo aun muy limitado en América del Sur a pesar del potencial de la región para este tipo de investigaciones. Sin embargo, en la última década se han incrementado los proyectos etnoarqueológicos, sobre todo por parte de los arqueólogos sudamericanos. En este artículo se revisan las bases conceptuales de esta subdisciplina y se analizan sus tendencias en América del Sur. Se discute el potencial de la Etnoarqueología, no solo para vincular la conducta humana con los derivados materiales, sino tambien para entender bajo que condiciones es esperable cierto tipo de registro arqueológico Por último, se explora la utilidad de la Etnoarqueología para abordar otras formas de pensamientos y de patrones de racionalidad, que aunque difíciles de indentificar en el registro arqueológico, son claves para entender aspectos esenciales de las sociedades del pasado.

Palabras clave: América del Sur, etnoarqueología, América del Sur.

Abstract: Despite all of the potential that South America has for ethnoarchaeological research, the development of Ethnoarchaeology is still very limited in this region. Only in the last decade ethnoarchaeological projects have grown, specially among South American archeologists. The present article revises the conceptual foundations of this field of study and analyses its present tendencies in South America. A discussion of the field's potential is made, not only as to what it can bring to the study of the relationship between human conduct and material derivations, but also as to the conditions in which we can expect to find certain types of archeological data. At last, the present article explores the uses that can be made out of Ethnoarchaeology in the study of other forms of thought and other patterns of rationality - forms and patterns that are hard to be identified in

\footnotetext{
*Agradecimientos: Al Lic. Mariano Bonomo por su lectura crítica y sugerencias. A Clara Scabuzzo por su colaboración en la edición. Este trabajo es parte del Programa de Investigaciones INCUAPA (Investigaciones Arqueológicas y Paleontológicas del Cuaternario Pampeano) de la Universidad del Centro de la Pcia. de Buenos Aires.
}

Horizontes Antropológicos, Porto Alegre, ano 8, n. 18, p. 61-91, dezembro de 2002 
archeological data but are essential to understanding fundamental aspects of past societies.

Keywords: archeological theory, ethnoarchaeology, South America.

En este trabajo me propongo resumir las bases conceptuales y metodológicas de la Etnoarqueología y explorar algunas vías de aplicación que han sido poco desarrolladas en América del Sur. La Etnoarqueología ha sido mirada con cierta desconfianza por algunos arqueólogos debido a las dificultades que existen en extrapolar la información actualística hacia las sociedades del pasado a partir de que no están suficientemente desarrolladas las bases epistemológicas acerca de cómo hacer esta transmisión información, lo que genera dudas con respecto a la argumentación analógica. Esta cuestión generó un prolongado debate hace más de dos décadas entre aquellos que estaban en contra del uso de la argumentación analógica en la interpretación del registro arqueológico (Gould, 1980; Wobst, 1978) y los que la consideraban un elemento central en el proceso de inferencia en arqueología (Binford, 1967; Watson, 1979). Actualmente y sobre todo luego del análisis epistemológico llevado a por Wylie (1982, 1985), la inmensa mayoría de los arqueólogos reconocen la utilidad de la argumentación analógica en el proceso de interpretación o explicación del registro arqueológico y la consideran como indispensable (Hernando, 1995, p. 20; Sillar, 2000, p. 8). Además, ha sido también propuesto que la reacción de Gould no fue realmente en contra del uso de la argumentación analógica y que el mismo basa su discusión en asunciones uniformitaristas (Kuznar, 1995, 2001). A pesar de esto, no todos los arqueólogos están convencidos de la utilidad de la analogía etnográfica y de los mecanismos usualmente empleados para su aplicación (Gosden, 1999, p. 9).

Otro punto que ha generado desconfianza es que en menor o mayor grado, las sociedades indígenas actuales, que forman la fuente de la analogía, han tenido contacto con la cultura occidental y están integradas de una manera u otra al proceso de "globalización" (Burch; Ellana, 1994; Stahl, 1993; Wilmsen, 1989). En base a esta situación, que en la mayoría de los casos es absolutamente real, se ha planteado que las sociedades presentes no pueden servir de referentes análogo de las sociedades pasadas. Esta crítica es sin embargo injustificada por que, como se desarrollara en este trabajo, la investigación etnoarqueológica opera bajo los principios de la 
argumentación analógica y por lo tanto, los dos elementos de la analogía (la fuente y el sujeto) no deben ser iguales (en cuyo caso no sería necesario un razonamiento analógico) sino que deben tener ciertas condiciones de comparabilidad. La fortaleza de la analogía generada a partir de la Etnoarqueología no reside en el grado de semejanza entre la fuente (en este caso, la sociedad presente) y el sujeto (la sociedad pasada percibida a través del registro arqueológico) sino en la estructura lógica de la argumentación y en la similitud entre los términos de la relación. Obviamente, cuanto mayor es la semejanza entre la fuente y el sujeto, la argumentación analógica tiene un mayor potencial, pero este grado de semejanza por sí mismo no garantiza de modo alguno la fortaleza de la argumentación ni la veracidad de los enunciados.

Un tercer punto que ha producido cierta reticencia con respecto a la Etnoarqueología es que ha sido cuestionada desde el punto de vista ético, tanto por antropólogos como por arqueólogos. En una ocasión en 1991 un profesor de Antropología de la Universidad Nacional de Bogotá expresó en una reunión de Departamento su rechazo hacia la etnorqueología pues dijo que no sabía mucho de esta sub-disciplina, pero que no le parecía bien "ir a molestar a los indios vivos para entender los que le pasaba a los indios muertos". De manera más formal Gosden ha planteado también objeciones éticas muy fuertes:

I feel that ethnoarchaeology is immoral, in that we have no justification for using the present of one society simply to interpret the past of another.... Societies ought to be studied as interesting in their own right or not at all. (1999, p. 9).

Estos planteamientos extremos son absolutamente incorrectos. La arqueología, así como la etnografía o cualquier otra rama de las ciencias antropológicas tiene como uno de sus fines principales estudiar la variabilidad de las sociedades humanas y entender los procesos culturales, por lo tanto es totalmente lícito estudiar sociedades presentes ya sea para aportar de manera directa a estos fines o de forma más indirecta mediante la identificación de referentes análogos que sirvan para entender las sociedades del pasado. De una manera u otra las sociedades del presente son el recurso más importante para la antropología y expresar que pueden ser 
estudiadas "as interesting in their own right or not at all" implica una segmentación artificial, e incorrecta, en la producción del conocimiento antropológico y asumir, erróneamente, que las sociedades no son interesantes por "derecho propio" cuando se estudian desde la Etnoarqueología. Como se vera más adelante, las múltiples dimensiones de sociedades humanas, y no solo sus restos materiales, tienen cada vez más un papel central en la Etnoarqueología moderna. Por último, la subdisciplina actúa con sociedades vivas y debe sujetarse a las mismas estrictas reglas éticas de la etnografía contemporánea (David; Kramer, 2001, p. 84-89) y no desarrollar ningún tipo de actividad o investigación sin el consentimiento de los actores sociales involucrados.

Cuando se recurre a los modelos generados desde la Etnoarqueología habitualmente se restringe su uso a la configuración del registro en relación a los aspectos tecnoeconómicos de las sociedades del pasado. Sin embargo, como se desarrollará en este trabajo, creo que el enfoque etnoarqueológico tiene un potencial significativamente más amplio que ha sido subvalorado, sobre todo en América del Sur, en donde la existencia de una gran variedad de sociedades indígenas y la abundante información etnográfica y etnohistórica, favorecen el desarrollo de esta subdisciplina. En las páginas siguientes se analizará cómo la información etnoarqueológica puede ser de extrema utilidad para la interpretación del registro arqueológico desde varias perspectivas y se discutirá como aprovechar más y mejor su potencial en América del Sur.

\section{Antecedentes}

En primer lugar, es importante reconocer que el intento de usar la información etnográfica para interpretar el registro arqueológico no es nuevo y mucho menos es patrimonio exclusivo de la Etnoarqueología (David; Kramer, 2001; Oswalt, 1974). Lo que sí es novedoso es que la información etnoarqueológica haya sido obtenida por arqueólogos con el propósito central de ayudar a la comprensión del registro arqueológico. Jesse W. Fewkes en 1900 fue el primero usar la palabra para referirse a sus intentos de identificar en los yacimientos Hopi asociados por los Hopi actuales a determinados ritos (Stiles, 1977). Simultáneamente, los etnógrafos comenzaron a interesarse por los objetos cotidianos de las soci- 
edades nooccidentales. Entre estos, Pitt Rivers fue uno de los impulsores en dar cabida a la cultura material en los estudios antropológicos a principios de siglo (Oswalt, 1974 ). También Marcel Mauss tuvo un papel importante pues puso a los estudios de cultura material en un plano central de la investigación etnográfica; cuando se refirió a las dificultades de la encuesta etnográfica, propuso a la colección de objetos como un de las maneras de superarlas: "El objeto es en muchos casos, la prueba mejor de un hecho social; y un catalogo de instrumental mágico es uno de los mejores medios para elaborar una buena clasificación de ritos" (Mauss, 1971, p. 15) En América del Sur otros investigadores también lo hicieron muy tempranamente como por ejemplo Theodoro Koch-Grünberg en sus investigaciones entre los indígenas Amazónicos durante 1902 y 1903 (Koch-Grünberg, 1995). Es importante remarcar que en América del Sur hubo todo una tradición etnográfica austro-alemana que prestó mucha atención a la cultura material y realizó detalladas colecciones de objetos junto a datos detallados sobre su uso (Gusinde, 1982; Schmidt, 1914). La inmensa mayoría de esta información esta actualmente desaprovechada por los arqueólogos y los objetos recogidos descansan en los museos sin ser estudiados sistemáticamente desde una perspectiva contemporánea.

Oswalt y VanStone (1967) usaron nuevamente el nombre Etnoarqueología para referirse a la información oral sobre la cultura material en un yacimiento esquimal ocupado entre 1840 y 1910. En general entre 1956 y fines de los 60' se observa un creciente uso de la información etnográficas con fines de interpretar mejor el registro material del pasado y se comienzan a efectuar las primeras recolecciones de datos actuales desde una mirada arqueológica (David; Kramer, 2001). En este tiempo aún no había investigadores que se llamaran a sí mismos etnoarqueólogos.

Con el advenimiento de la llamada "arqueología procesual" fue Binford (1967) quien comenzó a interesarse en el tema de manera sistemática en la analogía etnográfica en su trabajo pionero Smudged pit and hide smoking: the use of analogy in the archaeological reasoning. Posteriormente, Binford (1978) desarrolló teórica y conceptualmente su enfoque etnoarqueológico en Nunamiut Ethnoarchaeology. Estos aportes junto con los de Yellen (1977) y Gould (1978a, 1980) sentaron las bases metodológicas de la Etnoarqueología dentro del paradigma procesual y 
transformaron a la subdisciplina en una de las productoras más importantes de modelos para alimentar la "teoría de rango medio".

La Etnoarqueología contemporánea surgió como resultado directo de la valoración de los estudios actualísticos y del optimismo en el potencial de estos para explicar el registro arqueológico. Es por eso que partir de finales del '70 y sobre todo, desde los ' 80 comenzaron los estudios específicos sobre sociedades vivas efectuados por arqueólogos (Kent, 1984; Watson, 1979). De esta forma se planteaba algo novedoso que era buscar principios generales que conectaran el comportamiento humano con la cultura material y obtener conclusiones que no dependieran exclusivamente del campo teórico de la antropología socio-cultural. El optimismo inicial de la arqueología procesual en la creencia de que la conducta humana estaba sujeta a leyes (mas o menos similares a las de la biología) impregnó a la Etnoarqueología y dirigió el debate conceptual de esta en los años 70’. Durante estos primeros años de la subdisciplina, también existía el convencimiento subyacente de que se podrían generar leyes universales que relacionaran la conducta humana con los restos materiales (Yellen, 1977) y de hecho se supuso que junto con la arqueología experimental sería la principal fuente para la producción de esta leyes (Schiffer, 1978).

A comienzos de los 80’, la Etnoarqueología amplió su enfoque y comenzó también a ser desarrollada desde el postprocesualismo, pero de manera distinta (David; Sterner; Gavua, 1988; Hodder, 1982, 1991). En parte, estos nuevos desarrollos ya habían sido anticipados por Gould (1978b). Desde el postprocesualismo se expande el rango de interés de la Etnoarqueología sobre todo por que amplía su orbita mas allá de los aspectos tecnoeconómicos y apunta al entendimiento de niveles mayores de complejidad, intentando discernir los correlatos materiales de los aspectos cognitivos. sociales e ideológicos. Dentro de este nuevo marco conceptual la Etnoarqueología no se interesa por "explicar" en el sentido procesual del término (o sea entender la observación o proceso como resultado de una ley o a algún otro modelo teórico universal) sino que trata de interpretar el significado para la sociedad que lo produjo. De esta manera se enfatizaron algunos aspectos poco tratados en las investigaciones previas tales como el estudio simbólico y el de las dimensiones noutilitarias de la cultura material dentro de la trama social (ver por ejemplo los trabajos reunidos en Hodder, 1982). 
Ya en la década de los 90' los estudios etnoarqueológicos se multiplicaron y abordaron el estudio de todo tipo de sociedades (ver resumen en David; Kramer, 2001). Las preguntas se diversificaron y en todas las grandes regiones del mundo se montaron proyectos etnoarqueológicos de largo alcance. En esta década se evidencian claramente las dos posiciones de las décadas anteriores y aunque los enfoques postprocesuales se van popularizando, un importante número de etnoarqueólogos considera que la producción de leyes o "lawlike propostions" son objetivos posibles y necesarios para la arqueología y la Etnoarqueología (Kuznar, 1995; O'Connell, 1995).

Paradójicamente, en la medida que se incrementa el interés por este tipo de estudios y se valoriza su aporte para el proceso de inferencia arqueológica, cada vez mas disminuyen las sociedades "tradicionales" y en conse-cuencia se reduce significativamente el rango de variación de los referentes análogos. Como expresé al principio, la fortaleza de la argumentación analógica no reside en el grado de aislamiento de la sociedad usada como fuente. Sin embargo, la riqueza de la Etnoarqueología está en la captación de las variaciones de las condiciones culturales (tecnoeconómicas, sociales e ideacionales) de producción material de manera de poder identificar esta variación en el registro arqueológico. En consecuencia, la occidentalización de las sociedades indígenas disminuye notablemente la posibilidades de disponer de referentes análogos contemporáneos que puedan reflejar algunas de las condiciones de las sociedades pasadas o que sean comparables en algunos términos.

\section{Que es y para que sirve la etnoarqueologia?}

La Etnoarqueología ha sido entendida y definida de varias maneras y se han propuesto también otros vocablos como sinónimos tales como "arqueología viva", "etnografía arqueológica" o "arqueoetnografía". Las primeras definiciones la consideraban como la comparación entre los datos arqueológicos y etnográficos (Gould, 1978; Stiles, 1977). Para Stanislawsky (1977), la Etnoarqueología es la colección de información etnográfica original para ayudar interpretar a la información arqueológica y para Steensberg (1980; Ravn, 1993) es el uso controlado de la información etnográfica para explicar el registro arqueológico. Actualmente se cuenta 
con una variedad de definiciones (ver resúmenes en David; Kramer, 2001, p. 12); entre las mas simples están la de MacEachern (1996, p. 245) que propone que es "... la intesección de gente viviente y las construcciones arqueológicas" o a la de Hanks (1983, p. 351) que expresa que es "la aplicación de métodos arqueológicos a los datos etnográficos". Entre las definiciones más complejas se destaca la de David, una de mis preferidas, quien expresa que

la Etnoarqueología incluye el campo de estudio de la producción, tipología, distribución, consumo, y descarte de la cultura material, con especial referencia a los mecanismos que relacionan variabilidad y la variación al contexto sociocultural y a la inferencia de los mecanismos de procesos del cambio cultura. (1992)

Otra definición interesante es la de Sillar quien propone que la Etnoarqueología debe ser

the study of how material culture is produce, used and deposit by contemporary societies in relation to the wider social, ideological, economic, environmental and/or technical aspects of the society concerned, and with specific reference to the problems of interpreting archaeological material. (2000, p. 6)

En general las definiciones hacen rígidos a conceptos que deberían tener algo de flexibilidad, pero tienen un carácter operativo y comunicacional innegable. Teniendo esto en cuenta esta condición y basándome en el uso y desarrollo corriente de la disciplina, mi propia definición, flexible y abierta, expresa que la etnoaqueología es una subdisciplina de la arqueología y de la antropología social que obtiene información sistemática acerca de la dimensión material de la conducta humana, tanto en el orden ideacional como en el fenomenológico (en le sentido de Goodenough, 1964). Es una generadora de referentes analógicos para la interpretación arqueológica y es una fuente de producción y testeo de hipótesis y modelos acerca de cómo funcionan las sociedades Además, como expresó Kuznar (2001, p. 4) la etnarqueología es uno de las mejores vías para proveer la información requerida para contextualizar las analogías y para justificar asunciones. 
En general, la Etnoarqueología es entendida hoy en día como una subdisciplina de la arqueología (David; Kramer, 2001), a lo que yo agregaría también de la antropología sociocultural. Ambas disciplinas pueden ser consideradas "madres" de la Etnoarqueología, aunque es obvio que el desarrollo de esta se da casi exclusivamente dentro del campo de la arqueología. Esto no sólo esta relacionado a que los arqueólogos son quienes hacen Etnoarqueología, sino que son casi los únicos que la consumen. Lamentablemente, los antropólogos socioculturales aun no han incorporado a su debate el enorme potencial que tiene la información generada desde esta subdisciplina y la siguen considerando "cosa de arqueólogos".

En su uso corriente es considerada como una metodología de obtención de datos de sociedades vivas, pero desde una perspectiva arqueológica y sobre todo, prestando especial atención a los derivados materiales de las conductas humanas. Por lo tanto, esta estrategia de investigación implica trabajo de campo, fundamentalmente (aunque no exclusivamente) mediante la observación participante. Esta condición sería importante para discernir entre lo que se llama actualmente Etnoarqueología y lo que sería otro tipo de estrategia de investigación, tal como el uso de datos etnográficos o etnohistóricos en algún paso de la interpretación del registro arqueológico. Aunque las fronteras entre estas dos estrategias no forman una línea sólida, yo creo junto con otros etnoarqueólogos (David; Kramer, 2001) que la segunda debe quedar por ahora fuera del campo de la Etnoarqueología ya que no implica trabajo de campo con sociedades vivas, lo que es para mi uno de los componentes esenciales de la subdisciplina. Sin duda, los datos provenientes de investigaciones etnográficas (stricto sensu) y de los documentos históricos son de gran utilidad para la interpretación arqueológica y pueden servir para la formulación y el testeo de hipótesis así como para proveer fuentes de analogía, pero en la casi totalidad de los casos carecen de información sistemática y controlada sobre los productos materiales de las conductas a la que hacen referencia. Por lo tanto, no consideraré dentro de esta revisión a algunas investigaciones de América del Sur, que se presentan como etnoarqueológicas, pero que a pesar de manejar información interesante y de contribuir al conocimiento sobre los grupos indígenas de la región, no aportan datos de trabajos de campo originales sobre poblaciones vivas. Me estoy refiriendo al grupo de trabajos generados por el equipo de 
arqueólogos catalanes que desde 1986 esta investigando en Tierra del Fuego (Estévez; Vila, 1996). La estrategia de investigación utilizada es el uso complementario de fuentes etnohistóricas y de información arqueológica pero no la generación de modelos que articulen las conductas de sociedades contemporáneas con sus derivados materiales y con la interpretación del registro arqueológico.

Hay por lo menos tres campos en donde la etnoaqueología tiene utilidad para interpretación arqueológica. Por supuesto, estos campos no son compartimentos cerrados ya que se encuentran interconectados con amplias interfaces. Estos son:

1. Buscar relaciones recurrentes entre la conducta humana y cultura material.

Este es el sentido más usual dentro de la Etnoarqueología contemporánea y es probablemente dentro de este campo se inscriben los objetivos de la inmensa mayoría de los proyectos actuales. Este objetivo esta relacionado directamente con la construcción de la teoría de rango-medio en el sentido de Binford (1983, p. 14) "para establecer relaciones no-ambiguas de causa-efecto entre los causales dinámicos y los derivados estáticos". Las investigaciones sobre uso y descarte de artefactos; matanza, transporte y consumo de presas, construcción y abandono de viviendas o secuencias de fabricación de objetos que se han llevado a cabo en América del Sur se enmarcan dentro de este campo (García,1988; Jones, 1993; Stahl; Zeidler, 1990, Yacobaccio; Madero, 1994)

2. Generar modelos y proponer sus derivados materiales contextualizados dentro de los ordenes social e ideacional, abordando sistemas mas complejos.

En esta caso la Etnoarqueología apunta a la comprensión de la conducta humana contextualizada dentro de las esferas social e ideacional y apunta a entender los condicionantes culturales específicos de cada sociedad. Desde esta aplicación la Etnoarqueología no sólo aborda la relación entre la conducta humana y sus derivados materiales en situaciones en la cuales las variables tengan un control ajustado, sino que integra esto a niveles más complejos de las sociedades cuya dimensión material no es tan directa y su detección no es obvia (por ejemplo la relación entre los recursos y la movilidad o entre el parentesco y la producción cerámica) (Holster, 1996; Nielsen, 1997). Los recientes estudios etnoarqueológicos sobre la religión andina de Kuznar (2001) pueden también ejemplificar este campo. 


\section{Para entender y explorar otras formas de pensamiento}

Dentro de este campo se busca abordar patrones de racionalidad diferentes al occidental. En esta última aplicación de la Etnoarqueología la correlación con cultura material pasa a un segundo plano ya que se pretende entender otras formas de pensamiento y lógicas diferentes, más allá de sus correlatos materiales (Hernando, 1995). Este tipo de aplicación se basa la asunción de que determinados patrones de racionalidad del presente pueden entregar claves para entender como operaban algunos de estos en el pasado. Obviamente no se trata de entender en profundidad pautas de pensamiento ya extinguidas, pero si de detectar algunas claves de su funcionamiento $\mathrm{y}$ de discernir en los casos que sea posible, como y que factores ideológicos y sociales (además de los tecnoeconómicos) actuaron en la configuración del registro material. Estos objetivos han motivado, con variado éxito en los resultados obtenidos, las investigaciones de Delfino (2001) (David; Sterner; Gavua, 1988; Haber, 2001; Hernando, 1995; Hodder, 1982; Politis, 1996a; 1999; Sillar, 2000). Obviamente, dentro es dentro de este campo de aplicación donde las bases metodológicas están menos desarrolladas y se encuentran en una fase exploratoria.

Además de estos tres campos de aplicación, que insisto no se comportan como campos aislados, un servicio principal de la etnoaqueo-logía es sensibilizar a los arqueólogos (los cuales pertenecen a nuestra sociedad occidental y desarrollan su vida cotidiana en un medio urbano) hacia otras formas de pensamiento y de conceptualización de la realidad, que aunque distintas, tienen algunos elementos comunes con las sociedades indígenas del pasado. De esta manera, mas allá de la información etnoarqueológica que un investigador pueda obtener en el campo, la experiencia etnográfica permite ampliar el horizonte creativo del arqueólogo, el cual se constituye en una herramienta poderosa para interpretar el registro arqueológico. Este servicio de la arqueología ha sido ya reconocido por varios investigadores (David, 1992) y muy recientemente ha sido también remarcado, con un alto grado de optimismo para la arqueología andina

I am wholly convinced that in areas like the Andes the critical use of local analogies ('direct historical analogy' either from the ethnographic present or the historical record) is essential if archaeologists are to try to avoid the bias of uncritically imposing western attitudes on the Andean past. (Sillar, 2000, p. 8). 
Por ultimo, un servicio significativo de la Etnoarqueología ha sido el de contribuir a la reconceptualización de la cultura material. Este aporte, junto con la importante producción francesa en teoría social han sido los pilares que fundaron los enfoques modernos de la arqueología postprocesual (David; Kramer, 2001, p. 410).

\section{La Etnoarqueologia en America del Sur}

La Etnoarqueología es una subdisciplina que comenzó en América del Sur casi simultáneamente que en el resto del mundo, aunque inicialmente fueron realizados por extranjeros. Los primeros estudios sistemáticos se realizaron en los 70’ y el antecedentes pionero es quizás el trabajo de Lyon (1970) sobre la destrucción de huesos por perros en una aldea de la Amazonia Peruana. Desde sus fases iniciales, los trabajo se concentraron en dos regiones y en temas determinados. Uno de estos fue el uso y descarte de la alfarería en las tierras bajas de Ecuador, especialmente entre los Shipibo-Conibo (DeBoer, 1974; DeBoer; Lathrap, 1979); estos trabajos fueron iniciados por D. Lathrap quien presentó los primeros resultados en las reuniones anuales de la Society of American Archaeology y luego fueron continuados por J. Zeidler (1984) quien llevo a cabo una parte etnoarqueológica dentro su tesis doctoral. Otro de las areas/temas de interés inicial fue la Etnoarqueología de los pastores de camélidos Andinos (Horn, 1984; Miller, 1977). Entre los estudios etnoarqueológicos tempranos de arqueólogos locales se destacan los de I. Wüst quien hizo un estudio de la fabricación de alfarería de los Carajá de Aruanã y luego utilizó los datos para analizar los materiales arqueológicos de una antigua aldea Carajá (Wüst, 1975). En esta contribución Wüst se basó en los principios de la analogía histórica o directa. También se destacan los estudios de T. Miller Jr. quien realizó interesantes observaciones sobre la talla lítica entre los últimos Xetá del estado de Paraná en Brasil (Miller Jr., 1975, 1979). Este autor efectuó además algunas reflexiones tempranas sobre la Etnoarqueología y alertó sobre el potencial de las sociedades indígenas de América del Sur (Miller Jr., 1981-1982). Observaciones mas completas sobre los Xetá fueron luego publicadas por A. Laming-Emperaire junto a M.J. Menezes y M. D. Andreatta (Laming-Emperaire; Menezes; Andreatta, 1978). 
Hacia fines de los $80^{\prime}$ se multiplican los temas y las áreas de interés etnoarqueológico en América del Sur y varios arqueólogos sudamericanos comienzan a desarrollar proyectos etnoarqueológicos de largo alcance. Este nuevo conjunto de investigaciones se llevó a cabo desde diferente marcos conceptuales y apuntó a problemas diversos. Dentro de los estudios etnoarqueológicos en la región se perfilan tres tendencias. Una es la que restringe los casos de estudio a los efectos físicos de conductas definidas dentro de sistemas con variables que puedan ser, en principio, mejor controladas, como por ejemplo la producción de cerámica (Cremonte, 1988-89; García, 1988; 1993), el desmembramiento de las carcasas (Jones, 1983; 1993), la recolección de raíces silvestres (Greaves, 1996), la acumulación diferencial de basura en los pisos de las viviendas y en los asentamientos (Nasti, 1993; Stahl; Zeidler, 1990), la estructura los campamentos temporarios y residenciales entre foragers (Borrero; Yacobaccio, 1989; Greaves, 2000; Jones, 1996; Politis, 1995) y aldeanos horticultores (Assis, 1995, p. 96) o la relación entre las presas de caza y la tecnología del arco y flecha (Greaves, 1997). También se han abordado temas que involucran variables mas complejas, especialmente en la Etnoarqueología de pastores andinos (Kuznar, 1995; Tomka, 1993; 2001) tales como el relevamiento de dos grupos de variables: los patrones de movilidad y asentamiento y el manejo y explotación ganadera (Caracotche, 2001; Ventura; Belardi, 2001; Yacobaccio; Madero, 1994; Yacobaccio; Madero; Malmierca, 1998). Dentro de esta tendencia, se ha propuesto que el esfuerzo debe estar dirigido a incluir los casos particulares en modelos teóricos generales que sean universales (Yacobaccio, 1995). En su mayoría este grupo de trabajos enfatiza los aspectos tecnofuncionales de la cultura material y las determinantes ecológicas de la conducta humana. Esta tendencia está fuertemente influida por el procesualismo y se podría incluir dentro de lo que Hodder (2002) ha denominado la tendencia analítica, que tiene al trabajo de Binford (1978) como el principal referente.

Por otro lado, la segunda tendencia esta orientada hacia el estudio de sistemas más complejos, donde las variables son más difíciles de controlar, pero que dan cuenta de fenómenos más diversos e intenta discernir, además de los aspectos tecnoeconómicos, los significados sociales e ideacionales de los objetos desde casos de estudio etnoarqueológicos. Se busca producir conocimiento sobre las relaciones generales entre el comportamiento huma- 
no y sus derivados materiales. Dentro de esta tendencia se encuentran los estudios de la alfarería y la cestería como vehículos de expresión social (Silva, 2000; Holser, 1996; Sillar, 2000), la explotación de la fauna, y su consecuente formación de depósitos óseos, en función de los tabúes alimenticios (Politis; Martínez, 1996; Politis; Saunders, 2002), el análisis del ceremonialismo de los caravaneros andinos dentro del sistema de movilidad y asentamiento y la identificación de los diversos aspectos de la macroorganización del tráfico (Nielsen, 1997; 1997-98), la modificación del paisaje para la creación de espacios simbólicos (Dillehay, 1998), los aspectos émicos de la domesticación en los Andes (Haber, 2001) y los componentes simbólicos y sociales de la vivienda y el asentamiento en la Puna Meridional Argentina (Delfino, 2001). Para la mayoría de los etnoarqueólogos ubicados dentro de esta segunda tendencia, los artefactos (latu sensu) no son simplemente "cosas en sí mismas" sino que son consideradas como la representación de las ideas (Leach, 1977, p. 16). Es decir, son analizados como objetos polisémicos y polifuncionales, que operan simultáneamente en diferentes dimensiones (Dobres, 2001; Pfaffenberger, 1992). A esta segunda tendencia podría ser incluida dentro de lo que Hodder (2002) llamo la variante hermenéutica de la Etnoarqueología. Este enfoque es hermenéutico por que intenta elucidar el significado mediante la interpretación y apunta al entendimiento de un evento desde el punto de vista del actor, o desde "adentro" de una sociedad particular (Gadamer, 1976).

Dentro de ambas tendencias hay también variaciones importantes y por supuesto no a todos los proyectos en cuestión se le aplican las características enunciadas. Ambas están atadas a los efectos materiales de la conductas y a las propiedades de estos (densidad, variabilidad, distribución, etc), pero mientras que la primera intenta establecer relaciones no ambiguas y regularidades transculturales entre las conductas y sus derivados materiales, la segunda apunta pretende establecer relaciones de tipo general e intenta entender bajo que condiciones sociales se pueden esperar cierto tipo de registro arqueológico. La tendencia analítica busca identificar los principios generativos del registro, asumiendo que estos pueden ser ahistóricos y trans-culturales. El enfoque hermenéutico no busca identificar generalizaciones transculturales, utiliza con mayor énfasis y confianza la analogía continua o histórica y aunque reconoce las ventajas establecer 
algún tipo de generalizaciones, duda de que esto sea posible. Esta tendencia revaloriza la utilidad de las particularidades contextuales específicas y explora la continuidad del significado y la cosmovisión vinculado a símbolos e íconos específicos (Saunders, 1998; Wheatly, 1998). Como ejemplo de esto último, se pueden mencionar los estudios de Dillehay $(1990,1998)$ sobre el nguillatún de los mapuches de Chile. Dillehay analizó profundamente el sentido social y la cosmología reflejada en el diseño del campo del nguillatún, el que esboza el perfil del cuerpo del jaguar. En base a una serie de evidencias de distinto rango, el autor vincula el patrón en U del campo de nguillatún con el diseño de los centros ceremoniales del período Formativo Andino proponiendo que "aunque la cultura mapuche y las culturas formativas están alejadas unas de otras en el tiempo y en el espacio, ambas reflejan patrones socioculturales similares en el diseño y uso del espacio ceremonial y la iconografía" (Dillehay, 1990, p. 111). Este autor también discute la persistencia de los motivos felínicos, especialmente del jaguar, y la trascendencia en tiempo y en espacio de este referente simbólico que tuvo una función comunicativa importante en la sintaxis ideológica de las comunidades andinas.

Con respecto al abordaje de la obtención e interpretación de la información, la tendencia analítica tiene un enfoque mas etic (Harris, 1980), mientras que la hermenéutica se orienta hacia la búsqueda de lo émic. Obviamente, los aspectos émic de los significados de las conductas son difíciles de acceder, sobre todo teniendo en cuenta que los etnoarqueólogos no suelen pasar extensos períodos de campo a la manera de los estudios etnográficos clásicos. Sin embargo, esta dificultad técnica no debe servir de excusa para intentar descifrar los códigos simbólicos contenidos en las conductas y en su dimensión material. Es posible abordar estos incluso con estadías no demasiado prolongadas si es que diseñan estrategias específicas para obtener este tipo de información (Frias, 1993; Haber, 2001; Politis; Saunders, 2002; Silva, 2000).

Entre la Etnoarqueología de América del Sur, la perspectiva analítica ha sido la mas explorada, tanto en sociedades-cazadoras recolectores, como en grupos agrícolas y pastoriles. El segundo enfoque es más restringido y en el caso de sociedades cazadoras-recolectoras temas tales como la tabúes alimenticios y la existencia de lugares sagrados no han sido ni siquiera considerados como posibles agentes que contribuyen a la formación y 
distribución del registro arqueológico en América del Sur (para excepciones ver por ejemplo Politis; Martínez, 1996). Entre los caravaneros andinos, los estudios de Nielsen (1997-98) para buscar la dimensión material del ceremonialismo caravanero, los de Dillehay (1998) para la identificación de la construcción de espacios sociales y sagrados o los Frías (1993) para discernir el significado social e identatario de los motivos de la alfarería de los Piapoco son también ejemplos significativos.

La tercera tendencia, esta representada por un grupo de proyectos de investigación, sobre todo en Brasil, que busca obtener información etnoarqueológica para reconstruir el procesos histórico de grupos indígenas contemporáneos (Heckenberger; Peterson; Neves, 1999; Wüst, 1998; Wüst; Barreto, 1999). Esta tendencia busca entender los procesos de continuidad y cambio en contextos sociales específicos, mediante el uso complementario de la información etnográfica. Etnohistórica y arqueológica. De esta manera se propone que la continuidad cultural de la secuencia cronológica desde tiempos pre-hispánicos hasta el presente, en base a un "marcado conservatismo" no sólo en la organización espacial de las aldeas, sino también en la tecnología cerámica, la subsistencia y la localización de los asentamientos, "permits fairly detailed direct historical comparisons" (Heckenberger; Peterson; Neves, 1999). De alguna manera, este tipo de Etnoarqueología esta íntimamente ligado a lo que podríamos llamar `historia indígena' (Eremites de Oliveira, 2001) Aunque el carácter histórico-cultural de este enfoque podría reducir su aplicabilidad, el potencial de estas investigaciones para la interpretación arqueológica es enorme.

\section{Una propuesta para la etnoarqueologia de America del Sur}

En cualquiera de tres tendencias apuntadas, la Etnoarqueología aparece como uno de los programas mas firmes para la construcción de un cuerpo conceptual y metodológico que permita interpretar y explicar el registro arqueológico a través de enunciados testeables. Dentro de la tendencia analítica, puede producir un aporte sustantivo para la teoría de rango medio, o sea para generar modelos y recursos interpretativos que permita realizar inferencias acerca del registro arqueológico mediante la observación directa de sociedades contemporáneas. Desde la perspectiva hermenéutica, la Etnoarqueología se puede transformar también en una de las herramientas 
mas poderosas para abordar las dimensiones materiales de patrones de pensamientos diversos y para construir marcos interpretativos que sirvan para explorar lo social y lo ideacional en el regitro arqueológica. Desde la tercera perspectiva, la Etnoarqueología puede proveer correlatos detallados ya que obtiene información etnográfica de "grano fino" y la articula con los datos arqueológicos locales. Desde esta perspectiva se pueden ajustar muy claramente los límites de aplicación de la inferencia analógica ya que existe un control muy estricto entre los componentes de la fuente y el sujeto hacia donde se proyecta la información actual.

Sin embargo, el potencial de la etnorqueología, como expresé al comienzo del artículo esta subvalorado y subutilizado. La Etnoarqueología puede ser una herramienta que permita ir mas allá de los que se llama la "escalera de las inferencias de Hawkes" (1954) y que tanta influencia ha tenido en la arqueología contemporánea. Esta metáfora reflejaba la idea de que la arqueología debía estar restringida a la interpretación de los aspectos ambientales, tecnológicos y eventualmente sociales, ya que estos serían los únicos posibles de acceder con cierto grado de probabilidad. Hoy sabemos que esto no es así, ya que todas las actividades humanas derivan de patrones cognitivos que determinan las conductas técnicas mas simples, tales como cortar carne de una presa o tallar un raspador, hasta las más complejas como mantener una red de intercambio basada en mecanismos de reciprocidad entre parientes. Como todas estas tienen una dimensión material, las propiedades del registro arqueológico dependerán de la cosmovisión de los actores sociales que la produjeron. En otras palabras, el patrón de trozamiento, la forma de consumo y el lugar de descarte de un conjunto de huesos no sólo estará influenciado por las características de las presas y las necesidades energéticas de los cazadores y sus familias, sino también por el orden ideacional de estos y por su contexto histórico. Al respecto Sillar ha llegado a conclusiones similares:

The method of killling and butchering an animal is dependant of many different factors such as how hunting parties are organised, how these relates to domestic organization, the way caribou meat is commonly prepared, who eats it, the ideological significance of the animal, the historical importance of hunting to the group and recent process of social change, etc. Only in-depth work which relates the 
hunting to much wider aspects of the society can hope to understand why the animal was butchered in any specific way. (2000, p. 7).

En principio, la tendencia hermenéutica sería una herramienta eficaz por que permite producir enunciados acerca de aspectos simbólicos o ideacionales de las sociedades del pasado. Esto ha sido fuertemente criticado desde el neopositivismo, pero como lo han expresado Preucel y Hodder (1996), el enfoque hermenéutico es inherente a la argumentación arqueológica y el interés por recobrar el significado (tanto de un objeto como de una acción o una conducta) es un aspecto central de la arqueología contemporánea, independientemente de que esté en cuestión la economía, el parentesco o la regligión En este sentido entonces, lo que todos los arqueólogos hacen es entonces una forma de hermenéutica (Preucel; Hodder, 1996) y lo que varía es entonces es hacia que esfera de la cultura esta dirigida.

Propongo que el diseño de programas de Etnoarqueología, para alcanzar el máximo de su potencial (o sea que no se quede solamente en aspectos tecno-económicos), debería tener en cuenta los siguientes principios, tanto en la diagramación de las estrategias de investigación, como en la obtención de los datos y en su interpretación.

a. Las sociedades indígenas, tanto actuales como precolombinas, construyen su realidad de forma diferente a la occidental y con diferentes parámetros (Hernando, 2002) Para la construcción de esta realidad generan referentes simbólicos con una dimensión material. "La concepción del mundo depende de la construcción simbólica de la realidad, la cual es percibida por los actores sociales en contextos socioculturales e históricos específicos" (Grebe, 1995-96, p. 137).

b. Los artefactos arqueológicos son objetos polisémicos y un análisis de ellos debería intentar contemplar su funcionamiento en tantas dimensiones como sea posible.

c. El entendimiento profundo de los significados de las conductas y de sus correlatos materiales es un aspecto de muy difícil acceso desde el registro arqueológico. Sin embargo, es posible entender cómo y bajo que sistemas simbólicos (u ordenes ideacionales o cosmovisiones) están operando. Mediante las representaciones icónicas, la tradición oral, la historia y la mitología indígena, se han preservado y comunicado patrones 
cognitivos y simbólicos que dan cuenta de algunas ideas centrales y principios dominantes de las sociedades precolombinas.

En primer termino, se debe reconocer que aún esta operando la idea, errónea, de que se debe buscar primero las relaciones eco-utilitarias que ocurren en las situaciones bajo observación y ver hasta que punto la variación en la conducta observada puede ser explicada por esta circunstancias inmediatas (Gould; Watson, 1982, p. 67). Bajo esta perspectiva, solo si se ha agotado este nivel de explicación sin un resultado satisfactorio se puede pasar a un nivel más alto "namely the ideational realm of shared traditions" (Gould; Watson, 1982, p. 67). Desde mi punto de visto todos estos niveles de explicación deberían ser abordados simultáneamente ya que no hay una conducta 'eco-utilitaria', desprovista de una contexto social, de una dimensión simbólico y que no sea la expresión fenomenológica de un orden ideacional. Todos estos generadores y condicionantes de la conducta, los técnicos, los económicos, los sociales y los ideacionales, operan simultáneamente. Por lo tanto, se deben desarrollar estrategias de investigación etnoarqueológicas diseñadas específicamente para recuperar los múltiples significados de los objetos y las diversas causales de la conducta humana. Es decir no se debería priorizar las funciones tecnoeconómicas de los objetos (lo que Pfaffenberger llamó la "Visión Standard de la Tecnología") ni asumir como únicas o primarias las causales tecno-ambientales (lo que se ha llamado funcionalismo ecológico). En algunas proyecto etnoarqueológicos de América del Sur esto ha sido explicita y sistemáticamente incorporado (Delfino, 2001; Dillehay, 1998; Politis, 1998; 1999; Sillar, 2000; Silva, 2000).

En segundo lugar, para los efectos de la interpretación de los referentes simbólicos, se puede considerar metodológicamente adecuado, rescatar la decodificación ofrecida por algunas fuentes documentales y por los testimonios de los significados y las causas emic en las sociedades indígenas actuales (Grebe, 1995-96). Esto es lo que provee la Etnoarqueología hermenéutica y es la línea argumental seguida por varios arqueólogos para interpretar el significado de objetos o estructuras que operaron primariamente en el plano de lo ideológico y lo simbólico (Falchetti, 1999; ReichelDolmatoff, 1988; Velandia, 1994; 1999). Es cierto que esto tiene dos problemas importantes. Uno es que los símbolos culturales son arbitrarios y que el significado de los objetos y de los elementos naturales varia de una 
cultura a otra de manera aparentemente azarosa (Layton, 1992, p. 212). El segundo es que el significado asociado a íconos o a símbolos puede cambiar a través del tiempo (Saunders, 1998) o ser alterado por las transformaciones que sufre una sociedad y su contexto tanto natural como político-social. Es por eso que proponer recuperar el significado profundo de los símbolos o de objetos cotidianos que también tuvieron una dimensión social o ideacional es actualmente ilusorio. Lo que si es posible es identificar algunas claves bajo las cuales operaron y detectar algunas de las variables socio-ideacionales que afectaron la vida de los artefactos mientras estaban circulando en la cultura viva. Además, el simbolismo asociado a ciertos objetos o íconos en la arqueología americana que ha sobrevivido hasta nuestros días, aunque obviamente transformado, y ha sido registrado desde los primeros momentos del contacto. Esto ha permitido proposiciones muy interesantes para rastrear su significado en momentos precolombinos (Legast, 1998; Saunders, 1998; Tochetto, 1996). En otros casos, la organización de ciertos elementos en sociedades actuales daría pistas para abordar sus múltiples significados en el pasado. Por ejemplo, cuando Nielsen discute la función y la detección a arqueológica de los sitios rituales de los caravaneros, reconoce que no sería posible a través de estos sitios acceder al universo simbólico del pasado pero a la vez sostiene que "su rígida organización interna, localización y la naturaleza de los desecho asociados, permitirían quizás, identificar arqueológicamente la presencia de estructuras recurrentes en la conducta ritual, delineando sus rasgos fundamentales y sus variaciones en el tiempo y en el espacio" (Nielsen, 1997-98, p. 172).

Es obvio que a medida que ascendemos en los niveles de complejidad y abstracción, disminuye la capacidad de control de la variables en juego y la fortaleza de las relaciones entre la conducta y sus derivados materiales. Sin embargo ¿es esto una barrera para tratar de abordar sistemáticamente el estudio de los sistemas sociales y de creencias, para identificar en la sociedades presentes principios generativos particulares y que puedan ser testeados con el registro arqueológico? Para sociedades con cierta complejidad y jerarquización esto es habitual (Falchetti, 1999; González, 1977; 1992; Grebe, 1995-96; Reichel-Dolmatoff, 1988; Velandia, 1994) ya que se asume que por ejemplo la producción de objetos de carácter simbólico y la construcción de monumentos son inherentes a partir de cierto 
grado de complejidad social y como consecuencia se espera un registro arqueológico que de cuenta de esto. Sin embargo, estas sociedades tanto como las igualitarias (cazadores-recolectores) o de bajo nivel de complejidad o jerarquización no sólo producen objetos, consumen alimentos o construyen viviendas exclusivamente como consecuencia de la adaptación al medio ambiente natural y social. Sin duda, como lo enseña la etnografía y la etnohistoria, tanto los cazadores-recolectores como pastores, las sociedades aldeanas simples o los señoríos mantienen sistemas de creencias y reglas sociales complejos que permean en todas sus actividades y que determinan por ejemplo, que animales y que partes se van a consumir, donde se instala el campamento, de que lugar se traerán las rocas para confeccionar artefactos o cómo se distribuye el excedente de producción. De la misma manera, usan y jerarquizan el espacio no solo en función de la estructura de los recursos, son también a la percepción simbólica del paisaje, la cosmovisión y a la trama social de los territorios (ver por ejemplo un caso de aplicación arqueológica para América del Sur en Curtoni, 2000). Toda esta percepción y conceptualización del paisaje (que determina que lugares usar, como y para que) tiene una dimensión material que, aunque de manera incompleta y fragmentaria, es posible abordar desde la etnorqueología y la arqueología. Por ejemplo, los pastores del Departamento de Lípez (Sur de Bolivia) así como de otros lugares del altiplano ordenan el espacio en relación a referentes naturales y simbólicos y estas concepciones "se ponen de manifiesto en la organización regular de la conducta en múltiples contextos, como la disposición de los edificios y altares en el espacio doméstico, las ceremonias y la organización de las jaras" (Nielsen, 1997-98, p. 174).

Estos aspectos sociales e ideológicos han sido severamente subestimados en la arqueología y la Etnoarqueología de América del Sur, especialmente en sociedades sin jerarquías o de baja complejidad y han estado afuera de la agenda de investigación regional, tanto de científicos locales como extranjeros. Por supuesto, la re-orientación de las estrategias de investigación etnoarqueológica no implica abandonar los detallados análisis de los aspectos estrictamente tecnoutilitarios de la conducta. Ellos han demostrado de ser de extrema utilidad y en muchas casos un excelente punto de partida (pero no de llegada). Sin embargo, su alto valor descriptivo y diagnóstico no invalida el hecho de que el abordaje de exclusivo de estos 
aspectos deja afuera, durante el proceso de interpretación importantes componentes de la conducta humana que tienen una dimensión material. Además, los resultados de estos análisis, van perdiendo fortaleza en la medida que mecánicamente se transforma una correlación entre variables en una explicación. Los índices de utilidad económica de las presas o los estudios acerca de los componentes y las técnicas de la manufactura cerámica son y serán probablemente vitales para seguir avanzando en el entendimiento del registro arqueológico de América del Sur. Pero su utilidad será mucho mayor si los podemos integrar en modelos más comprensivos que los vinculen con la trayectoria histórica y con la esfera social e ideacional.

\section{Referencias}

ASSIS, V. S. Um Estudo da Casa Mbya pela Perspectiva Etnoarqueológica. Coleção Arqueologia. Porto Alegre, ano 2, n.1, p. 519-26, 1995-96.

BINFORD, L. Smudge pits and hide smoking. The use of analogy in archaeological reasoning. American Antiquity, n. 32, p. 1-12, 1967.

BINFORD, L. Nunamiut ethnoarchaeology. New York: Academic Press, 1978.

BINFORD, L. Working at archaeology. New York: Academic Press, 1983.

BORRERO, L.; YACOBACCIO, H. Etnoarqueología de Asentamientos Aché. Journal De La Societé Des Américanistes, LXXV, p. 7-33, 1989. BURCH JR., E; ELLANA, L. Introduction. In: BURCH Jr., E; ELLANA, L. (Ed.). Key issue in hunter- gatherer research. Oxford: Berg Publishers, 1994. p. 1-10.

CARACOTCHE, M. S. The Invisibility of Time: An Ethnoarchaeological Study of the Temporary Sites of Herders of the Southern Puna. In: KUZNAR, L. (Ed.). Ethnoarchaeology of Andean South America. Michigan, International Monographs in Prehistory, Ethnoarchaeological Series. n. 4, p. 97-115, 2001.

CREMONTE, B. Técnicas alfareras tradicionales en la Puna: Inti-Cancha. Arqueología Contemporánea. ano 2, n. 2, p. 5-29, 1988-89. 
CURTONI, R. Archaeological Approach to the Perception of Landscape and Ethnicity in the West Pampean Region, Argentina. 1999. Dissertation (Master)-Institute of Archaeology, University of London, London, 2000.

DAVID, N. Integrating ethnoarchaeology: a subtle realist perspective. Journal of Anthropological Archaeology. n. 11, p. 330-359, 1992.

DAVID, N.;KRAMER, C. Ethnoarchaeology in Action. Cambridge: Cambridge University Press, 2001.

DAVID, N.; STERNER, J.; GAVUA, K. J. Why pots are decorated? Current Anthropology, n. 29, p. 365-389, 1988.

DEBOER, W. R. Ceramic longevity and archaeological interpretation: an example from the Upper Ucayali, Peru. American Antiquity, n. 39, p. 335344, 1974.

DEBOER, W.; LATHRAP, D. The Making and Breaking of Shipibo-Conibo Ceramics. In: KRAMER, C. (Ed.). Ethnoarchaeology: Implications of Ethnography for Archaeology. New York: Columbia University Press, 1979. p. 102-138.

DELFINO, D. Of Pircas and the Limits of Society: Ethnoarchaeology in the Puna, Laguna Blanca, Catamarca, Argentina. In: KUZNAR, L. (Ed.). Ethnoarchaeology of Andean South America. Michigan, International Monographs in Prehistory, Ethnoarchaeological Series 4, 2001. p. 97-137.

DILLEHAY, T. Araucanos: Presente y Pasado. Santiago de Chile: Editorial Andres Bello, 1990.

DILLEHAY, T. Felines, patronyms and history of the Araucanians in the Southern Andes. In: SAUNDERS, N. (Ed.). Icons of Power: Feline Symbolism in the Americas. London: Routledge, 1998. p. 203-228.

DOBRES, Marsha A. Technology and Social Agency. Great Britain: Blackwell Publishers, 2000.

ESTÉVEZ, J.; VILLA, A. Etnoarqueología: el nombre de la cosa. In: ESTÉVEZ, J.; VILLA, A. (Org.). Encuentros en los conchales fueginos. Bellaterra: CSIC-UAB, 1996. p. 17-24.

FALCHETTI, A. M. El poder simbólico de los metales: la tumbaga y las transformaciones metalúrgicas. Boletín de Arqueología, Santafé de Bogotá: Fundación de Investigaciones Arqueológicas Nacionales, ano 14, n. 2, p. 53-82, 1999. 
FRIAS, I. Ajuar cerámico de los Piapoco: un caso de estilo como transmisor de información. In: FERNANDEZ, Francisco; GRASSON, Rafael (Ed). Contribuciones a la Arqueología Regional de Venezuela. Colinas de Bello Monte: Fondo Editorial Acta Científica Venezolana, 1993. p. 107-138.

GADAMER, H. G. On the scope and function of hermeneutic reflection. In: LINGE, D. (Ed.). Philosophical Hermeneutics. Berkeley, 1976.

GARCIA, L. C. Etnoarqueología: Manufactura de Cerámica en Alto Sapagua. In: YACOBACCIO, H. (Org.). Arqueología Contemporánea Argentina. Buenos Aires: Editorial Búsqueda, 1988. p. 33-58.

GOSDEN, C. Anthropology and Archaeology: A changing relationship. London: Routledge, 1999.

GONZÁLEZ, A. R. Arte Precolombino de la Argentina: Introducción a su historia cultural. Buenos Aires: Filmediciones Valero, 1977.

GONZÁlEZ, A. R. Las placas metálicas de los Andes del Sur: Contribución al estudio de las religiones precolombinas. Mainz am Rhein: Verlag Phillipp von Zabern, 1992.

GOODENOUGH, W. H. Introduccion. In GOODENOUGH, W. H (Ed.). Explorations in cultural anthropology. New York: McGraw Hill, 1964. p. 1-24. GOULD, R. The anthropology of human residues. American Anthropologist, n. 80, p. 815-835, 1978a.

GOULD, R. From Tasmania to Tucson: new directions in ethnoarchaeology. In: GOULD, Richard (Ed.). Explorations in ethnoarchaeology. Albuquerque: University of New Mexico, 1978b. p. 1-10.

GOULD, R. Living Archaeology. New York: Cambridge University Press, 1980 GOULD, Richard; WATSON, Patty Jo. A dialogue on the meaning and use of analogy in ethnoarchaeological reasoning. Journal of Antrhopological, n. 1, p. 355-381, 1982.

GREAVES, R. Ethnoarchaeology of wild root collection among savanna foragers of Venezuela [Paper presented at the $54^{\text {th }}$. Annual Plains Anthropological Conference, October 31, Iowa City, Iowa]. 1996.

GREAVES, R. Hunting and Multifunctional Use of Bows and Arrows. Ethnoarchaeology of Technological Organization among the Pumé Hunters of Venezuela. In: KNECHT, H. (Ed.) Projectile Technology. New York: Plenum Press, 1997. p. 287-320. 
GREAVES, R. Forager Landscape Use and Residential Organization [Paper presented at the $65^{\text {th }}$. Annual Meeting of the Society for American Archaeology, April 5-9, Philadelphia, Pennsylvania]. 2000.

GREBE, M. E. Continuidad y cambio en las representaciones icónicas: significado simbólico sur-andino. Revista Chilena de Antrpología, ano 13, p. 137-153, 1995-96.

GUSINDE, M. Los Indios de Tierra del Fuego. Buenos Aires: CAEA, 1982. HABER, A. Observations, Definitions and Pre-understanding in the Ethnoarchaeology of Pastoralism. In: KUZNAR, L. (Ed.). Ethnoarchaeology of Andean South America, International Monographs in Prehistory, Ethnoarchaeological Series 4, Michigan, 2001. p. 31-37.

HANKS, C. An Ethnoarchaeological approach to the seasonality of Historic Cree sites in Central Québec. Arctic, n. 36, p. 350-355, 1983.

HARRIS, M. Cultural Materialism: The Struggle for a science of culture. New York: Vintage Books, 1980.

HAWKES, C. Archaeological theory and method: some suggestions from the Old World. American Anthropologist, n. 56, p. 155-168, 1954.

HECKENBERGER, M.; PETERSON, J.; NEVES, E. G. Village size and permanence in Amazonia: two archaeological examples from Brazil. American Antiquity, v. 10, n. 4, p. 353-376, 1999.

HERNANDO, A. La Etnoarqueología hoy: una vía eficaz de aproximación al pasado. Trabajos de Prehistoria, ano 52, n. 2, p. 15-30, 1995.

HERNANDO, A. Arqueología de la identidad. Madrid: Editorial Akal, 2002.

HODDER, I. Symbols in Action: Ethnoarchaeological Studies of Material Culture. Cambridge, 1982.

HODDER, I. Reading the Past: Current Approaches to Interpretation in Archaoelogy. Cambridge: Cambridge University Press, 1991.

HODDER, I. Archaeological theory. In: CUNLIFFE, Barry; DAVIES, Wendy; RENFREW, Colin (Ed.). Archaeology: The Widening Debate. Oxford: British Academy, 2002. p. 77-90.

HOLSTER, D. Technical choices, social categories and meaning among the Andean potter of Las Animas. Journal Of Material Culture, v. 1, n. 1, p. 63-92, 1996. 
HORN, D. Marsh Resource Utilization and the Ethnoarchaeology of the Uru-Muratos of Highland Bolivia. (Ph. Dissertation)-Washington University, St. Louis, 1984.

JONES, K. Forager Archaeology: The Aché of Eastern Paraguay. In: LEMOINE, G. M.; MACEACHERN A. S. (Ed.). Carnivores, Human Scavengers, and Predators: A Question of Bone Technology. University of Calgary: Archaeological Association, 1983. p. 171-191.

KENT, S. Analyzing Activity Areas. Alburquerque: University of New Mexico, 1984.

KOCH-GRÜNBERG, Theodoro. Zwei Jahre unter den Indianern: Reisen in Nordwest- Brasilien 1903/1905. Berlin, 1995. 2 v.

KUZNAR, L. A. Awatimarka: The Ethnoarchaeology of an Andean Herding Community. Forth Worth: Harcourt Brace College Publishers, 1995.

KUZNAR, L. A. Introduction. In: KUZNAR, L. (Ed.). Ethnoarchaoelogy of Andean South America. Michigan, International Monographs in Prehistory, Ethnoarchaeological Series 4, 2001. p. 15-18.

KUZNAR, L. A. An Introduction to Andean Religious Ethnoarchaeology: Preliminary Results and Future Directions. In: KUZNAR, L. (Ed.). Ethnoarchaoelogy of Andean South America. Michigan, International Monographs in Prehistory, Ethnoarchaeological Series 4, 2001. p. 38-66.

LAMING-EMPERAIRE, A.; MENEZES, M. J.; ANDREATTA, M. D. O trabalho da pedra entre os Xetá da Serra dos Dourados, Estado do Paraná. In: Coletânea de Estudos em Homenagem a Anette Laming-Emperaire. Coleção Museu Paulista, 1978. p. 19-82. (Série Ensaios, ano 2).

LAYTON, R. Ethnographic Analogy and the Two Archaeological Paradigms. In. GOLDSMITH, S. et. al. Ancient images, ancient thought: the archaeology of identity [Proceedings of the $23^{\text {rd }}$ Annual Chacmool Conference]. Calgary: Archaeological Association, University of Calgary, 1992. p. 211-221.

LEACH, E. A View from the Bridge. In: SRIGGS, M. (Ed.). Archaeology and Anthropology. Oxford: BAR Supplementary Series, 1977. p. 161-176. LEGAST, A. Feline symbolism and material culture in prehistoric Colombia. In: SAUNDERS, N. (Ed.). Icons of Power: Feline Symbolism in the Americas. London: Routledge, 1998. p. 122-124. 
LYON, P. Differential Bone Destruction: An Ethnographic Example. American Antiquity, n. 35, p. 213-215, 1970.

MACEACHERN, S. Foreign countries: the development of ethnoarchaeology in sub-Saharan Africa. Journal of World Prehistory, n. 10, p. 243-304, 1996.

MAUSS, M. Introducción a la Etnografía. Madrid: Ediciones ISTMO, 1971. MILLER, G. An introduction to the ethnoarchaology of Andean camelids. (Ph.D. Dissertation)-University of California, Berkeley, 1977. Unpublished. MILLER JR., T. Tecnologia litica arqueológica (Arqueología Experimental no Brasil). Anais do Museu de Antropologia, Florianópolis, t. 7, p. 5-135, 1975.

MILLER Jr., T. Stonework of the Xetá Indians of Brazil. In: HAYDEN, Brian (Ed.). Lithic use-wear analysis. New York: Academic Press, 1979, p. 401-409.

MILLER Jr., T. Etnoarqueologia: implicações para o Brasil. Arquivo do Museu de História Natural, v. 6-7, p. 293-310, 1981-1982.

NASTI, A. Etnoarqueología de los residuos humanos. Análisis de estructuras de sitios de asentamientos de pastores de la Puna Meridional Argentina. Arqueología, n. 3, p. 9-39, 1993.

NIELSEN, A. El tráfico caravanero visto desde La Jara. Estudios Atacameños, n. 14, p. 339-371, 1997.

NIELSEN, A. Tráfico de caravanas en el sur de Bolivia: observaciones etnográficas e implicancias arqueológicas. Relaciones de la Sociedad Argentina de Antropología, n. 22-23, p. 139-178, 1997-98.

OLIVEIRA, J. E. A história indígena em Mato Grosso do Sul, Brasil: dilemas e perspectivas. Revista Territórios e Fronteiras, ano 2, n. 2, p. 115-124, 2001.

OSWALT, W. Ethnoarchaeology. In: DONNAN, C. H.; CLEWLOW, C. W. (Ed.). Ethnoarchaeology. Los Angeles: Monograph IV. Institute of Archaeology, University of California, 1974. p. 3-14.

OSWALT, W. H.; VANSTONE, J. W. The ethnoarchaeology or Crow village, Alaska. Bulletin 199. Washington, D.C.: Bureau of American Ethnology, 1967. 
PFAFFENBERGER, B. Social Anthropology of Technology. Annual Review of Anthropology, ano 21, p. 491-516, 1992.

POLITIS, G. Moving to produce: Nukak mobility and settlement patterns in Amazonia. World Archaeology, ano 27, p. 492-510, 1996a.

POLITIS, G. Nukak. Santafé de Bogotá: Instituto SINCHI, 1996b.

POLITIS, G. Arqueología de la Infancia: una perspectiva etnoarqueológica. Trabajos de Prehistoria, ano 55, n. 2, p. 5-19, 1998.

POLITIS, G. Plant exploitation among the Nukak hunter-gatherers of Amazonia: between ecology and ideology. In: GOSDEN, C.; HATHER, J. (Ed.). The Prehistory of Food: Appetites for Change. London: Routledge, 1999. p. 99-126.

POLITIS, G.; MARTINEZ, G. La cacería, el procesamiento de las presas y los tabúes alimenticios. In: POLITIS, G. (Ed.). Nukak. Santafé de Bogotá: Instituto SINCHI, 1996. p. 231-80.

POLITIS, G.; SAUNDERS, N. Archaeological correlates of ideological activity: food taboos and spirit-animals in an Amazonian hunter-gatherer society. In: MIRACLE, P. (Ed.). Consuming Passions: Archaeological studies of material culture. Cambridge: Mc Donald Institute, 2002. p. 113130.

PREUCEL, R.; HODDER, I. (Ed.). Contemporary Archaeology in Theory. Oxford: Blackwell, 1996.

RAVN, M. Analogy in Danish Prehistory. Norwegian Archaeological Rewiev, ano 26, n. 2, p. 59-90, 1993.

REICHEL-DOLMATOFF, G. Orfebrería y chamanismo: Un estudio iconográfico del Museo del Oro. Medellín: Editorial Colina, 1988.

SAUNDERS, N. Architecture of Symbolism: The feline image. In: SAUNDERS, N. (Ed.). Icons of Power: Feline Symbolism in the Americas. London: Routledge, 1998. p. 12-52.

SCHIFFER, M. Methodological Issues in Ethnoarchaeology. In: GOULD, R. A. (Ed.). Explorations in ethnoarchaeology. Albuquerque: University of New Mexico Press, 1978. p. 229-247.

SCHMIDT, M. Die Guato und ihr Gebiet. Ethnologische und archaologische Ergebnisse der Expedition zum Caracara-fluss in Matto-Grosso. BaesslerArchiv Berlin, ano 4, n. 6, p. 251-283, 1914. 
SILLAR, B. Shaping Culture. Making pots and Constructing Households: An Ethnoarchaeological Study of Pottery Production: Trade and Use in the Andes. Oxford: BAR International Series 883, 2000.

SILVA, F. A. As Tecnologías e Seus Significados: Um Estudo da cerámica dos Asuriní do Xingu e da cesteria dos Kayapó-Xikrin sob uma Perspectiva Etnoarqueológica. Tese (Doutorado)-Faculdade de Filosofia e Ciencias Humanas, Universidade de São Paulo, São Paulo, 2000.

STAHL, A. Concepts of time and approaches to analogical reasoning in historical perspective. American Antiquity, n. 58, p. 235-260, 1993.

STAHL, P.; ZEIDLER, J. Differential bone-refuse. Accumulation in food preparation and traffic areas on an early Ecuadorian house floor. Latin American Antiquity, ano 1, n. 2, p. 150-169, 1990.

STANISLAWSKY, M. B. Ethnoarchaeology of Hopi and hopi Tewa pottery makin: styles of learning. In: INGERSOLL, D. T; YELLEN, J. E.; MACDONALD, W. (Ed.). Experimental archaeology. New York: Columbia University Press, 1977. p. 378-408.

STEENSBERG, A. New Guinea Gardens: A Study oif Husbandry with Paralells in Prehistoric Europe. London: Academic Press, 1980.

STILES, D. Ethnoarchaeology: A discussion of methods and applications. Man, ano 12, n. 1, p. 87-103, 1977.

TOMKA, S. Site abandonment behavior among transhumant agropastoralist: the effects of delayed curation on assemblages composition. In: CAMERON, C. M.; TOMKA, S. (Ed.). Abandonment of Settlement and Regions. Cambridge: Cambridge University Press, 1993. p. 11-24.

TOMKA, S. An Ethnoarchaeological Study of Tool Design and Selection in an Andean Agro-Pastorial Context. Latin American Antiquity, ano 12, n. 4, p. 395-412, 2001.

TOCHETTO, F. Possibilidade de Interpretação do conteúdo simbólico da arte gráfica Guaraní. Revista do Museu de Arqueologia e Etnologia, n. 6, p. 33-45. 1996.

VELANDIA, C. San Agustín: Arte, Estructura y Arqueología. Modelo para una Semiótica de la Iconografía Precolombina. Bogotá: Banco Popular: Universidad de Tolima, 1994. 
VELANDIA, C. The archaeological culture of San Agustín. Towards a new interpretation. In: POLITIS, Gustavo G.; ALBERTI, Benjamín (Ed.). Archaeology in Latin America. London: Routledge, 1999.

VENTURA, B.; BELARDI, J. B. When Clouds Cover the Woods: Ethnoarchaeology in the Yungas of Salta, Argentina. In: KUZNAR, L. (Ed.). Ethnoarchaeology of Andean South America. Michigan International Monographs in Prehistory, Ethnoarchaeological Series 4, 2001. p. 67-83.

WATSON, P. J. Archaeological ethnography in western Iran. Vicking Fundation Publications in Anthropology 57. Tucson: University of Arizona Press, 1979.

WHEATLY, D. Reader in archaeological theory: postprocessual and cognitive approaches. London, 1998.

WILMSEN, E. Land filled with Flies: A Political economy of the Kalahari. Chicago: Chicago University Press, 1989.

WOBST, H. M. The archaeo-ethnography of hunter-gatherers and the tyranny of the ethnographic record in Archaeology. American Antiquity, n. 43, p. 303-309, 1978.

WÜST, Irmhild. A Cerâmica Carajã de Aruanã. Goiânia. Anuario De Divulgação Científica, n. 2, ano 2, p. 96-165, 1975.

WÜST, I. Continuities and discontinuities: archaeology and ethnoarchaeology in the heart of the Eastern Bororo territory, Mato Grosso, Brazil. Antiquity, n. 72, p. 663-675, 1998.

WÜST, I.; BARRETO, C. The Ring Village of Central Brazil: A Challenge for Amazonian Archaeology. Latin American Antiquity, n. 10, p. 3-23, 1999.

WYLIE, A. An analogy by any other name is just as analogical: a commentary on the Gould-Watson dialogue. Journal of Anthropological Archaeology, ano 1, p. 382-401, 1982.

WYLIE, A. The reaction against analogy. Advances in Archaeological Method and Theory, n. 8, p. 63-111, 1985.

YACOBACCIO, H. Qué nos cuentan las cocinas. Etnoarqueología en Inca Cueva. Palimpsesto, ano 3, p. 133-38, 1993.

YACOBACCIO, $\mathrm{H}$. El aporte de la Etnoarqueología al conocimiento del registro arqueológico pastoril andino. In: CONGRESO NACIONAL DE ARQUEOLOGÍA CHILENA, HOMBRE E DESIERTO, 13. Actas... Antofagasta, Chile, v. 9, n. 1, p. 309-316, 1995. 
YACOBACCIO, H.; MADERO, C. (Ed.). Etnoarqueología de pastores surandinos: una herramienta para conocer el registro arqueológico. In: Jornadas de Arqueología e Interdisciplinas. CONICET-Programa de Estudios Prehistóricos. Buenos Aires, 1994, p. 203-236.

YACOBACCIO, H.; MADERO, C.; MALMIERCA, M. (Ed.). Etnoarqueología de Pastores Surandinos. Buenos Aires: Grupo Zooarqueología de Camélidos, 1998.

YELLEN, J. Archaeological approaches to the present. New York: Academic Press, 1977.

ZEIDLER J. Social space in Valdivia Society: Community patterning and domestic structure at Real Alto, 3.000-2.000 B.C. (Ph.D. Dissertation). Urbana, Illinois, 1984. Unpublished. 